\title{
Risk Factors of New Vertebral Compression Fracture After Percutaneous Kyphoplasty or Vertebroplasty: A Retrospective Study of 752 Patients
}

\section{Bo-bo Zhang}

First Affiliated Hospital of Xi'an Jiaotong University

Zhong-wei Song

First Affiliated Hospital of Xi'an Jiaotong University

\section{Ying Wang}

First Affiliated Hospital of Xi'an Jiaotong University

\section{Zhi-wei Ren}

First Affiliated Hospital of Xi'an Jiaotong University

\section{Wei-zhou Yang}

First Affiliated Hospital of Xi'an Jiaotong University

Yi-min Yang ( $\sim$ guke473@126.com )

First Affiliated Hospital of Xi'an Jiaotong University

\section{Research Article}

Keywords: New vertebral , compression fractures , PKP , PVP , Risk factor

Posted Date: November 19th, 2021

DOI: https://doi.org/10.21203/rs.3.rs-1057960/v1

License: (c) (i) This work is licensed under a Creative Commons Attribution 4.0 International License.

Read Full License 


\section{Abstract}

Summary Irregularly postoperative anti-osteoporosis treatment (AOT), number of treated vertebrae (NTV) $\geq 2$, bone mineral density (BMD) $\leq \bigotimes 3.0$ SDs, body mass index $(B M I)<18.5 \mathrm{~kg} / \mathrm{m}^{2}$ or $B M I \geq 24 \mathrm{~kg} / \mathrm{m}^{2}$, vertebral height loss ratio (VHLR) $₫ 20 \%$ were strong risk factors associated with new vertebral compression fractures (NVCF) after percutaneous vertebroplasty or kyphoplasty (PVP/PKP) in patients with osteoporotic vertebral compression fractures (OVCF). There was no difference between NVCF occurred adjacent or remote after PKP/PVP intervention.

Introduction NVCF have been connected to PVP/PKP surgery for patients with VCF. There are some debates about whether new vertebral body fractures are simply a result of the natural progression of osteoporosis or whether they should be regarded as a consequence of augmentation. We tried to investigate and identify the risk factors which may be relevant to NVCF after PVP/PKP surgery in OVCF patients.

Methods The authors retrospectively analyzed the occurrence of NVCF in 752 patients treated with PVP or PKP for OVCF. Possible risk factors, such as age, gender, refracture time (RT), AOT, NTV, BMD, BMI, and VHLR, were assessed.All methods are carried out in accordance with relevant guidelines and regulations.

Results Significant differences $(P<0.05)$ were found between the NVCF and control groups with regard to $\mathrm{AOT}$, number of treated vertebrae, BMD, BMI, and VHLR by univariate analysis. And irregularly AOT, NTV $\geq$

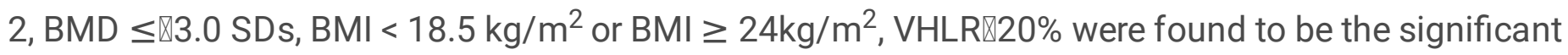
factors in multivariate analysis. To evaluate a direct and more precise effect of the procedures on untreated vertebrae, the NVCF group was subdivided into adjacent and remote fracture groups. All of factors have no significant difference between two subgroups.

Conclusion In the current study, the incidence of NVCF after PVP/PKP is $9.58 \%$. Irregularly AOT, NTV $\geq 2$,

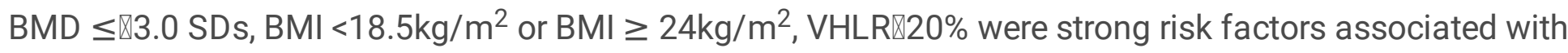
NVCF after PVP/PKP in patients with OVCF. There was no difference between NVCF occurred adjacent or remote after PKP/PVP intervention and that might be the result of the osteoporosis itself. All of those should be addressed during preoperative communication and postoperative management.

\section{Introduction}

As for the treatment of osteoporotic vertebral compression fractures (OVCF), percutaneous vertebroplasty (PVP) and percutaneous kyphoplasty (PKP) were proved to be safe and efficient [1]. The complication rate of PVP/PKP is reported to be low for the treatment of OVCF [2]. Some authors reported that NVCF is one of the complications associated with PVP/PKP, especially for adjacent vertebral, varying from 10 to $30 \%[3,4]$. Some debates are about whether NVCF are simply a result of osteoporosis or whether they are the consequence of augmentation [5]. Herein, this study focused on the occurrence of NVCF that followed PVP/PKP surgery. In addition, we tried to investigate and identify the risk factors which may be relevant to NVCF after PVP/PKP surgery in OVCF patients. 


\section{Methods}

\section{Study subjects}

From January 2010 to January 2017, 752 patients with osteoporotic thoracic or lumbar vertebral fractures were treated by PVP/PKP at The First Affiliated Hospital of Xi'an Jiaotong University. There were 442 female patients and 310 male patients. Diagnosis criteria for OVCF are as follows: (1) back pain are consistent with radiologic examination;(2) X-ray showing height loss or endplate fracture;(3) magnetic resonance imaging (MRI) showing vertebral marrow edema, or whole-body bone scan showing significant uptake at the fracture level. Exclusion criteria consisted of the following: (1) other types of vertebral compression fracture, such as myeloma, tumor; (2) previous spinal surgery; (3) incomplete clinical data; (4) follow-up time is less than 2 years.

\section{Surgical Technique}

All PVP and PKP were performed by one surgeon (M.Y.Y.), through either a unilateral transverse processpedicle process or bilateral transpedicular approach. After the local anesthesia, the 11-G needle were advanced into the vertebral body under X-ray guidance. A polymethylmethacrylate (PMMA, Mendec Spine Resin, Italy) was used as the bone filler during the PVP. In the PKP, we use the Kyphon ballon tamps (KMC Kyphoplasty System, Shanghai Kinetic). If leakage occurs when bone cement filling is not satisfactory, the following measures can be taken to continue the operation. Firstly, try to use secondary filling: wait for the bone cement to solidify and block the rupture, then fill it again. Secondly, adjust the position of the bone filler or use a side opening filler. Lastly, readjust the puncture direction or use contralateral puncture filling. Patients were encouraged to ambulate $4 \mathrm{~h}$ after the procedure.

\section{Data Collection}

Patients were followed-up at 1 day, 1 month, 3 months, 6 months during the first year after the operation, and then followed up every year. Patients were recommended to have a high-calcium diet, moderation exercise, and sun exposure 2 hours one day, calcium (600-1200 mg daily) and calcitriol (0.25-0.5ug daily) and alendronate medication (70 mg weekly). We measured the bone mineral density (BMD) annually, and the medication was continued at least three years if no side effects occurred. The diagnosis criteria of NVCF was the same to OVCF at the first time. When symptomatic NVCF occurred, repeated PVP/PKP or conservative treatment were carried out.

The following possible risk factors were collected: age, gender, refracture time (RT), postoperative antiosteoporosis treatment (AOT), number of treated vertebrae(NTV), lumbar spine BMD, body mass index (BMI), and preoperative vertebral height loss ratio (VHLR): (1- (anterior height of fractured vertebra / (anterior height of upper vertebra + anterior height of inferior vertebra) / 2))*100\%. The bigger VHLR implies a larger degree of wedge deformity. Symptomatic NVCF patients during the follow-up were 
grouped into the "NVCF" group. The others were grouped into the control group. The NVCF group was further divided into "adjacent" and "remote" groups.

\section{Statistical Analysis}

Data were analyzed by using SPSS 22.0 software. Data were presented as percentages for categorical variables. Differences between NVCF group and control group were assessed by using the chi-square test. Risk factors associated with NVCF were evaluated by using logistic regression analysis, and according to the inspection level, $\mathrm{P}<0.05$ was defined as statistically significant.

\section{Results}

No major complication was occurred after the PVP/PKP procedures. Among 752 patients who underwent PVP/PKP successfully, a total of 72 cases (28 male patients and 44 female patients) developed symptomatic NVCF and were classified in the "NVCF" group; this represented $9.58 \%$ of the patient population. The method of "Propensity Score Matching" has been applied to selected 72 patients without experience NVCF, who were considered as the control group.

Fifteen patients chose the conservative treatment. The other 57 patients selected repeated PKP/PVP for NVCF. Of all the 72 patients, thirty patients $(41.7 \%)$ had NVCF on the adjacent vertebra. Significant differences $(P<0.05)$ were found between the NVCF and control groups with regard to AOT, NTV, BMD, $\mathrm{BMI}$, and VHLR by univariate analysis (Table 1). Irregularly AOT, the number of treated vertebrae $\geq 2, \mathrm{BMD}$ $\leq \varangle 3.0 \mathrm{SDs}, \mathrm{BMI}<18.5 \mathrm{~kg} / \mathrm{m}^{2}$ or $\mathrm{BMI} \geq 24 \mathrm{~kg} / \mathrm{m}^{2}, \mathrm{VHLR} \otimes 20 \%$ were significant factors determined by multivariate analysis (Table 2). All the factors have no significant difference between the adjacent and remote fracture groups (Table 3 ).

\section{Table 1}

Summary of variables between the control and NVCF group following PVP/PKP for OVCF. 


\begin{tabular}{|c|c|c|c|}
\hline Variable & NVCF group $(n=72)$ & Control group $(n=72)$ & $P$ \\
\hline Gender & & & 0.7340 \\
\hline male & $28(38.9 \%)$ & $30(41.7 \%)$ & \\
\hline female & $44(61.1 \%)$ & $42(58.3 \%)$ & \\
\hline Age (years) & & & 0.7448 \\
\hline $60-70$ & $18(25.0 \%)$ & $16(22.2 \%)$ & \\
\hline $70-80$ & $36(50.0 \%)$ & $32(44.4 \%)$ & \\
\hline $80-90$ & $14(19.4 \%)$ & $18(25.0 \%)$ & \\
\hline$>90$ & $4(5.6 \%)$ & $6(8.3 \%)$ & \\
\hline AOT & & & $<.0001$ \\
\hline regular & $24(33.3 \%)$ & $50(69.4 \%)$ & \\
\hline irregular & $48(66.7 \%)$ & $22(30.6 \%)$ & \\
\hline NTV & & & 0.0247 \\
\hline 1 & $33(45.8 \%)$ & $20(27.8 \%)$ & \\
\hline$\geq 2$ & $39(54.2 \%)$ & $52(72.2 \%)$ & \\
\hline BMD (SDs) & & & $<.0001$ \\
\hline $\mathbb{\nabla} 83.0$ & $18(25.0 \%)$ & $56(77.8 \%)$ & \\
\hline$\leq \varangle 3.0$ & $54(75.0 \%)$ & $16(22.2 \%)$ & \\
\hline $\mathrm{BMI}\left(\mathrm{kg} / \mathrm{m}^{2}\right)$ & & & $<.0001$ \\
\hline $18.5 \rrbracket 23.9$ & $12(16.7 \%)$ & $48(66.7 \%)$ & \\
\hline$<18.5$ & $32(44.4 \%)$ & $13(18.1 \%)$ & \\
\hline$\geq 24$ & $28(38.9 \%)$ & $11(15.3 \%)$ & \\
\hline VHLR (\%) & & & $<.0001$ \\
\hline$\leq 20$ & $15(20.8 \%)$ & $41(56.9 \%)$ & \\
\hline$>20$ & $57(79.2 \%)$ & $31(43.1 \%)$ & \\
\hline
\end{tabular}

Table 2

The multifactor logistic regression analysis for the factors associated with NVCF after PVP/PKP. 


\begin{tabular}{|c|c|c|c|c|}
\hline Variable & Estimated value & $P$ & OR & $95 \% \mathrm{Cl}$ of $\mathrm{OR}$ \\
\hline AOT (irregular VS regular) & 2.04 & 0.0003 & 7.70 & $2.57,23.07$ \\
\hline NTV ( $\geq 2$ VS 1) & 1.62 & 0.0041 & 5.08 & $1.67,15.40$ \\
\hline 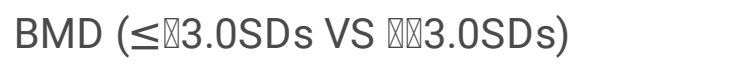 & 2.50 & $<.0001$ & 12.21 & $4.11,36.21$ \\
\hline $\operatorname{BMI}\left(<18.5 \mathrm{~kg} / \mathrm{m}^{2}\right.$ VS $\left.18.5 \llbracket 23.9 \mathrm{~kg} / \mathrm{m}^{2}\right)$ & 2.34 & 0.0004 & 10.42 & $2.84,38.13$ \\
\hline $\mathrm{BMI}\left(\geq 24 \mathrm{~kg} / \mathrm{m}^{2}\right.$ VS $\left.18.5 \bowtie 23.9 \mathrm{~kg} / \mathrm{m}^{2}\right)$ & 2.09 & 0.0008 & 8.08 & $2.37,27.58$ \\
\hline VHLR $(>20 \%$ VS $\leq 20 \%)$ & 1.76 & 0.0016 & 5.84 & $1.95,17.47$ \\
\hline
\end{tabular}

Table 3

Characteristics of patients in the adjacent NVCF group and remote NVCF group 
Variable

\begin{tabular}{|c|c|c|c|}
\hline & group $(n=72)$ & group $(n=72)$ & \\
\hline Gender & & & 0.4138 \\
\hline male & $10(33.3 \%)$ & $18(42.9 \%)$ & \\
\hline female & $20(66.7 \%)$ & $24(57.1 \%)$ & \\
\hline Age (years) & & & 0.4136 \\
\hline $60-70$ & $7(23.3 \%)$ & $11(26.2 \%)$ & \\
\hline $70-80$ & $16(53.3 \%)$ & $20(47.6 \%)$ & \\
\hline $80-90$ & $4(13.3 \%)$ & $10(23.8 \%)$ & \\
\hline$>90$ & $3(10.0 \%)$ & $1(2.4 \%)$ & \\
\hline Refracture time (months) & & & 0.9037 \\
\hline$\nabla 6$ & $4(13.3 \%)$ & $6(14.3 \%)$ & \\
\hline $6-12$ & $11(36.7 \%)$ & $13(31.0 \%)$ & \\
\hline $12-24$ & $12(40.0 \%)$ & $16(38.1 \%)$ & \\
\hline$\bigotimes 24$ & $3(10.0 \%)$ & $7(16.7 \%)$ & \\
\hline AOT & & & 0.1282 \\
\hline regular & $7(23.3 \%)$ & $17(40.5 \%)$ & \\
\hline irregular & $23(76.7 \%)$ & $25(59.5 \%)$ & \\
\hline NTV & & & 0.9045 \\
\hline 1 & $14(46.7 \%)$ & $19(45.2 \%)$ & \\
\hline$\geq 2$ & $16(53.3 \%)$ & $23(54.8 \%)$ & \\
\hline BMD (SDs) & & & 0.4076 \\
\hline $\mathbb{\square} \otimes 3.0$ & $9(30.0 \%)$ & $9(21.4 \%)$ & \\
\hline$\leq \bigotimes 3.0$ & $21(70.0 \%)$ & $33(78.6 \%)$ & \\
\hline BMI $\left(\mathrm{kg} / \mathrm{m}^{2}\right)$ & & & 0.6965 \\
\hline $18.5 \rrbracket 23.9$ & $4(13.3 \%)$ & $8(19.0 \%)$ & \\
\hline$<18.5$ & $15(50.0 \%)$ & $17(40.5 \%)$ & \\
\hline$\geq 24$ & $11(36.7 \%)$ & $17(40.5 \%)$ & \\
\hline VHLR (\%) & & & 0.1055 \\
\hline
\end{tabular}




\begin{tabular}{lll}
$\leq 20$ & $9(30.0 \%)$ & $6(14.3 \%)$ \\
\hline$>20$ & $21(70.0 \%)$ & $36(85.7 \%)$
\end{tabular}

\section{Discussion}

It is widely recognized that PKP/PVP can achieve satisfactory effect for the treatment of OVCF, which could relieve pain rapidly. However, unexpected NVCF sometimes occur after PKP/PVP, which needs repeated PKP/PVP as the treatment method. NVCF is a common complication of PVP/PKP that can cause acute back pain, limited daily activity, and then lead to patient dissatisfaction. Various studies have reported the NVCF rate after PVP/PKP ranged between 7.8-21.7\% [6-10]. In our study, it is larger in case population, and the NVCF rate of $9.58 \%$ is comparable to these data. Can NVCF be considered as a complication after PKP/PVP and which factor will cause it? Many authors proposed a lot of different risk factors for the NVCF after PKP/PVP [5, 11-13]. Therefore, it is important to know the variables that increase the risk of NVCF, then some strategies will be developed to minimize the risk. In our study, irregularly $\mathrm{AOT}, \mathrm{NTV} \geq 2, \mathrm{BMD} \leq-3.0 \mathrm{SDs}, \mathrm{BMI}<18.5 \mathrm{~kg} / \mathrm{m}^{2}$ or $\mathrm{BMI} \geq 24 \mathrm{~kg} / \mathrm{m}^{2}, \mathrm{VHLR} \otimes 20 \%$ were found to be the significant factors in multivariate analysis.

As a result of our study, BMD $\leq-3.0$ SDs is an important risk factor of new fractures after PKP/PVP and thus anti osteoporosis is important for prevention of NVCF. BMD can reflect the degree of osteoporosis. When T-score of BMD was less than -2.5 SDs, the osteoporosis can be diagnosed according to the WHO osteoporosis diagnosis principle [14]. Uppin et al. demonstrated that new fractures were more likely to develop as osteoporosis worsened [15]. Therefore, to a large extent, lower BMD may cause NVCF. Kaufman et al. had demonstrated that patients with fracture were closely related to low BMD and patients who suffered OVCF risk decline of $3 \%$ for each percentage point increase in BMD [16]. In our study, the BMD in NVCF group was significantly less than that in control group, which indicated that lower BMD was a risk factor of NVCF after PKP/PVP. AOT had significant difference in NVCF group and control group, and irregularly AOT was a risk factor for the NVCF. All of these illustrated that the improvement of bone quality was much important with regular medication to AOT. Vitamin $D$ insufficiency has been found to be a common but largely ignored health problem in China, VD insufficiency or deficiency, defined as low levels of serum 25-hydroxyvitamin D, which often coexists with osteoporosis [17]. So it is recommended that patients should have a high-calcium diet, moderation exercise, and sun exposure 2 hours one day, calcium (600-1200 mg daily) and calcitriol (0.25-0.5ug daily) and alendronate medication (70 mg weekly) to treat osteoporosis and achieve satisfactory results. Therefore, regular AOT and increased of BMD were important measures to reduce NVCF after PKP/PVP.

In one study, authors reported that BMI less than $22 \mathrm{~kg} / \mathrm{m}^{2}$ had a greater chance of developing NVCF after PKP/PVP [6]. The results of this study is consistent with the results mentioned above, in which BMI less than $18.5 \mathrm{~kg} / \mathrm{m}^{2}$ is significant on multivariate analysis. The reason may be that $\mathrm{BMI}$ is positively correlated with estrogen activity, and estrogen stimulates osteoblasts to increase bone mass by increasing osteoid secretion. However, the most interesting thing is that being overweight was found to 
increase the incidence of vertebral fracture in other literature [18], the reason may be these people lay much pressure on vertebral and easy to fracture. In the present study, the risk of new fractures increased both in the subgroup of $B M l<18.5 \mathrm{~kg} / \mathrm{m}^{2}$ and $B M I \geq 24 \mathrm{~kg} / \mathrm{m}^{2}$. Further studies are needed to identify the boundary at which BMI becomes a risk factor for NVCF after PVP/PKP.

In our study, the number of treated vertebral not less than two and VHLR>20 were considered to be the risk factor for NVCF after PVP/PKP. Delmas et al. found that the primary OVCF severity was the most important independent predictor of the risk for NVCF [19]. Ren HL et al. found that the number of initial vertebral fractures was an important risk factor for NVCF [20]. However, some studies showed that the NVCF after PVP/PKP was unrelated to the number of initial OVCF [21]. The present study confirmed that the greater the number of levels of initial OVCF, the higher the incidence of new fractures. Kang et al. found that a compression fracture with greater preoperative VHLR tend to increase the incidence of NVCF after PVP/PKP [22] and the result was consistent with ours. We think the reasons may be as follows: Firstly, not only the NTV $\geq 2$, but also VHLR $>20$ showed the osteoporosis were severity. The more severity of the osteoporosis, the more risk of NVCF. Secondly, with a large number of vertebrae fractures and greater VHLR, the overall kyphosis angle increases, leading to an increased load ' in anterior column and an increased risk of refracture.

Some researchers have found that approximately half of NVCF appeared in adjacent vertebrae among patients who underwent PVP/PKP [23]. Lo et al. reported that the proportion of new adjacent fractures was as high as $55.6 \%$ [24]. The reasons why NVCF occurred in adjacent vertebrae were as follows: some literature reveals that excessive volume of bone cement injection and uneven distribution under the endplate are likely to be potential risk factors for adjacent vertebral fractures [25]. Liebschner et al. believe that unilaterally distributed bone cement will cause uneven force inside the vertebral body, and the stress will shift to the unreinforced side, which may cause new vertebral fractures [26]. It is recommended that bilateral bone cement injection be used to achieve uniform stress distribution. One literature [27] reveals that under the same conditions, the sponge-like distribution form increases the contact area between the bone cement and the bone, and the load can be dispersed and transmitted through the bone cement. Although the mass-shaped bone cement has a volume-occupying effect, it is more conducive to the expansion of the fractured vertebra. However, the contact area between bone cement and bone is limited, which results in the localized conduction of local stress and increases the risk of re-collapse of fractured and adjacent vertebrae. Bone cement leak in the intervertebral space is a risk factor for adjacent vertebral fractures. The principle is that the bone cement leaking into the intervertebral space will produce a stiffness gradient, resulting in local high stress of the adjacent vertebral endplate, which is the "pillar effect". Trout et al. [28] reported that $41.4 \%$ of NVCF occurred in adjacent vertebrae and there was no difference between remote group and adjacent group. In our study, $41.7 \%$ of the NVCFs occurred at the adjacent vertebra, which were no difference compared with remote group. So, it is suggested that NVCF occurred adjacent or remote after PKP/PVP might be the result of the osteoporosis itself, and not due to the operation, if the PKP/PVP properly used. 
This study has several limitations. We included symptomatic fractures only, but the actual refracture rate would be higher. Another important limitation of this study is that it was a retrospective study, and some potential confounders may be neglected. Nonetheless, further multi-institutional, prospective, randomized, controlled studies are required to determine the true risks of NVCF after PVP/PKP.

\section{Conclusions}

In the present study, the incidence of NVCF after PVP/PKP is $9.58 \%$. Irregularly AOT, the number of treated vertebrae $\geq 2, \mathrm{BMD} \leq-3.0 \mathrm{SDs}, \mathrm{BMI}<18.5 \mathrm{~kg} / \mathrm{m}^{2}$ or BMI $\geq 24 \mathrm{~kg} / \mathrm{m}^{2}, \mathrm{VHLR}>20 \%$ were strong risk factors associated with NVCF after PVP/PKP in patients with OVCF. There was no difference between NVCF occurred adjacent or remote after PKP/PVP intervention and that might be the result of the osteoporosis itself. All of those should be addressed during preoperative communication and postoperative management.

\section{Declarations}

\section{Funding information}

This study was supported by the Shaanxi Province Natural Science Program (No. 2017JM8168 for Yi-Min Yang).

\section{Data availability}

The data sets generated and/or analyzed during the current study are not publicly available due to the need to protect patients' privacy but are available from the corresponding author upon reasonable request.

\section{Compliance with ethical standards}

\section{Ethics approval and consent to participate}

This study was approved by the ethics committees of the First Affiliated Hospital of Xi'an Jiaotong University, and the requirement of informed consent was waived owing to the retrospective nature of the study.

All methods are carried out in accordance with relevant guidelines and regulations.

\section{Conflict of interest}


None.

\section{References}

1. Yuan, W. H., Hsu, H. C. \& Lai, K. L. Vertebroplasty and balloon kyphoplasty versus conservative treatment for osteoporotic vertebral compression fractures: a meta-analysis. Med. (Baltim), 95 (31), e4491 (2016).

2. Guglielmi, G., Andreula, C., Muto, M. \& Gilula, L. A. Percutaneous vertebroplasty: indications, contraindications, technique, and complications. Acta Radiol, 46 (3), 256-68 (2005).

3. Ambrosanio, G. et al. Vertebroplasty in the treatment of spine disease. Interv Neuroradiol, 11 (4), 309-23 (2005).

4. Lavelle William, F. \& Cheney, R. (2006) Recurrent fracture after vertebral kyphoplasty. Spine J 6(5): 488-93. doi:10.1016/j. spinee. 2005. 10.013

5. Rho, Y. J., Choe, W. J. \& Chun, Y. I. Risk factors predicting the new symptomatic vertebral compression fractures after percutaneous vertebroplasty or kyphoplasty. Eur Spine J, 21 (5), 905-11. doi: (2012).

6. Lin, W. C. et al. New vertebral osteoporotic compression fractures after percutaneous vertebroplasty: retrospective analysis of risk factors. J Vasc Interv Radiol, 19 (null), 225-31. doi: (2008).

7. Syed, M. I. et al. (2005) New symptomatic vertebral compression fractures within a year following vertebroplasty in osteoporotic women.AJNR Am J Neuroradiol26(6):1601-4

8. Lee, W. S. et al. Risk factors of developing new symptomatic vertebral compression fractures after percutaneous vertebroplasty in osteoporotic patients. Eur Spine J, 15 (12), 1777-83 (2006).

9. Kim, S. H., Kang, H. S., Choi, J. A. \& Ahn, J. M. Risk factors of new compression fractures in adjacent vertebrae after percutaneous vertebroplasty. Acta Radiol, 45 (4), 440-5 (2004).

10. Moon, E. S. et al. The incidence of new vertebral compression fractures in women after kyphoplasty and factors involved. Yonsei Med. J, 48 (4), 645-52 (2007).

11. Chen, Z. Q., Wu, Y. P., Ning, S. H., Ma, T. X. \& Wu, Z. Y. Risk Factors of Secondary Vertebral Compression Fracture After Percutaneous Vertebroplasty or Kyphoplasty: A Retrospective Study of 650 Patients. Med. Sci. Monit, 25 (undefined), 9255-9261 (2019).

12. Feng, L., Feng, C., Chen, J., Wu, Y. \& Shen, J. M. The risk factors of vertebral refracture after kyphoplasty in patients with osteoporotic vertebral compression fractures: a study protocol for a prospective cohort study. BMC Musculoskelet Disord, 19 (1), 195 (2018).

13. Lee, D. G., Park, C. K., Park, C. J., Lee, D. C. \& Hwang, J. H. Analysis of Risk Factors Causing New Symptomatic Vertebral Compression Fractures After Percutaneous Vertebroplasty for Painful Osteoporotic Vertebral Compression Fractures: A 4-year Follow-up. J Spinal Disord Tech, 28 (10), E578-83 (2015).

14. Kanis, J. A., Melton, L. J., Christiansen, C., Johnston, C. C. \& Khaltaev, N. The diagnosis of osteoporosis. J. Bone Miner. Res, 9 (8), 1137-41 (1994). 
15. Uppin, A. A. et al. Occurrence of new vertebral body fracture after percutaneous vertebroplasty in patients with osteoporosis., 226 (1), 119-24 (2003).

16. Kaufman, J. M., Palacios, S., Silverman, S., Sutradhar, S. \& Chines, A. An evaluation of the Fracture Risk Assessment Tool (FRAX®) as an indicator of treatment efficacy: the effects of bazedoxifene and raloxifene on vertebral, nonvertebral, and all clinical fractures as a function of baseline fracture risk assessed by FRAX®. Osteoporos Int, 24 (10), 2561-9 (2013).

17. Lim, S. K., Kung, A. W. C., Sompongse, S., Soontrapa, S. \& Tsai, K. S. Vitamin D inadequacy in postmenopausal women in Eastern Asia. Curr Med Res Opin, 24 (1), 99-106 (2008).

18. Tanaka, S., Kuroda, T., Saito, M. \& Shiraki, M. Overweight/obesity and underweight are both risk factors for osteoporotic fractures at different sites in Japanese postmenopausal women. Osteoporos Int, 24 (1), 69-76 (2013).

19. Delmas, P. D. et al. Severity of prevalent vertebral fractures and the risk of subsequent vertebral and nonvertebral fractures: results from the MORE trial., 33 (4), 522-32 (2003).

20. Ren, H. L., Jiang, J. M., Chen, J. T. \& Wang, J. X. Risk factors of new symptomatic vertebral compression fractures in osteoporotic patients undergone percutaneous vertebroplasty. Eur Spine $J$, 24 (4), 750-8 (2015).

21. Komemushi, A. et al. Percutaneous vertebroplasty for osteoporotic compression fracture: multivariate study of predictors of new vertebral body fracture. Cardiovasc Intervent Radiol, 29 (4), 580-5 (2006).

22. Kang, S. K. et al. Predictive risk factors for refracture after percutaneous vertebroplasty. Ann Rehabil Med, 35 (6), 844-51 https://doi.org/doi:10.5535/arm.2011. (2011).

23. Chosa, K., Naito, A. \& Awai, K. Newly developed compression fractures after percutaneous vertebroplasty: comparison with conservative treatment. Jpn J Radiol, 29 (5), 335-41 (2011).

24. Lo, Y. P., Chen, W. J., Chen, L. H. \& Lai, P. L. New vertebral fracture after vertebroplasty. J Trauma, 65 (6), 1439-45. doi: (2008).

25. Cai, K. W., Lu, B., Luo, K. F. \& Jiang, G. Q. (2019) Progression of the risk factors researches of adjacent vertebral fractures after vertebral augmentation for osteoporotic vertebral compression fracture.Chin J Orthop39:1087-1095

26. Liebschner, M. A., Rosenberg, W. S. \& Keaveny, T. M. Effects of bone cement volume and distribution on vertebral stiffness after vertebroplasty. Spine, 26 (14), 1547-54 (2001).

27. Li, L. J. et al. Associated risk factors for cemented vertebral body re-wedge after percutaneous kyphoplasty. Chin J Trauma, 33 (8), 724 (2017).

28. Trout, A. T., Kallmes, D. F. \& Kaufmann, T. J. (2006) New fractures after vertebroplasty: adjacent fractures occur significantly sooner.AJNR Am J Neuroradiol27(1):217-23

\section{Supplementary Files}

This is a list of supplementary files associated with this preprint. Click to download. 
- additionaldocument.docx

Page 13/13 